\title{
Irrigation Control Through Acoustic Proximal Sensing of the Onset of Surface Water
}

\author{
Stuart Bradley 1, 2, *, Anna Radionova 3, Chandra Ghimire 4, Laura Grundy 5, Seth Laurenson 6, and \\ Val Snow ${ }^{7}$ \\ 1 Physics Department, University of Auckland, Auckland, New Zealand; s.bradley@auckland.ac.nz \\ 2 Inverse Acoustics Ltd., Auckland, New Zealand; inverse.acousticsl@gmail.com \\ 3 School of Physical Sciences, The University of Adelaide, Adelaide, South Australia; \\ anna.radionova@adelaide.edu.au \\ 4 AgResearch Ltd., Lincoln, New Zealand; Chandra.Ghimire@agresearch.co.nz \\ 5 AgResearch Ltd., Lincoln, New Zealand; Laura.Grundy@agresearch.co.nz \\ 6 AgResearch Ltd., Lincoln, New Zealand; Seth.Laurenson@agresearch.co.nz \\ 7 AgResearch Ltd., Lincoln, New Zealand; Val.Snow@agresearch.co.nz \\ * Correspondence: inverse.acoustics@gmail.com
}

\begin{abstract}
Irrigation is a useful crop enhancement procedure up to the point where free surface water appears. Thereafter, water can begin to flow into waterways, leaching nutrients and giving rise to environmental damage, as well as being a waste of a precious resource. The current work addresses the problem of measuring free water on the surface of agricultural soils by a real-time acoustic remote sensing method. Directional acoustic transmitter and receiver arrays are used to define a 'footprint' on the ground from which changes in reflectance are sensed. These arrays are mounted on a moving irrigator. Chirp signals are used to provide along-path resolution and to ensure robustness against unwanted acoustic background noise from farm machinery and the irrigator. Field measurements have been conducted above a well-defined 'quadrat' with controlled and measured water content, and also with the instrument mounted on an operational irrigator. A structured light camera mounted above the footprint is used to validate surface water fraction. It is found that the areal fraction of free water on the soil surface can be reliably estimated from changes in the amplitude of the reflected sound waves. The mechanism giving rise to the observed acoustic reflectivity changes is discussed and a model is developed which agrees with normalized intensity observations with a coefficient of determination $\mathrm{R}^{2}$ between 0.65 and 0.83 . The rms error between model predictions and observations is comparable to the rms variation of the measurements, indicating that there is insignificant error due to the choice of model.
\end{abstract}

Keywords: Surface water runoff; acoustic sensor array; reflected sound wave; reflection from rough surfaces

\section{Introduction}

The goal of the present work is to develop a relatively simple and accessible technology for real-time sensing of soil surface moisture, without requiring contact with the surface.

Smarter irrigation is one of many approaches to address increasing pressure on agriculture due to economic, land area, and production target challenges [1]. A by-product of increased irrigation arises when irrigation mobilizes pollutants and transports them to water bodies. Mobilization of pollutants particularly happens when the irrigation rate is greater than the critical rate at which the soil can accept water. This critical irrigation rate can change rapidly in time and space so good irrigation design can only partially address this issue. Currently, there are no useful sensors to 
provide real-time measurements of the soil surface conditions, so farmers cannot respond to the rapidly changing conditions by actively managing their irrigation.

The soil surface is naturally rough $[2,3]$ but will begin to smooth out as the small pockets of free water that develop normally during an irrigation event fill in some of the hollows in the surface. Free water is not bound to the soil surfaces but is instead free to flow directly into water bodies. When the pockets coalesce to form connected flow pathways [4-6] the free water can begin to move from the point of application. This is when water efficiency is lost, and environmental harm caused.

A number of studies have focused on the characterization of the speed and absorption of sound in different types of soils $[7,8]$ and a few attempts to characterize sound propagation through the soil with different moisture contents $[9,10]$, but these are in-situ rather than non-intrusive remote sensing or proximal sensing methodologies.

One potential method of sensing the nature of the soil surface is to use acoustic reflection. There is a substantial literature on soil reflectivity relating to outdoor sound propagation [11], although sound propagation studies are concerned with grazing incidence and large distances not relevant to close proximity sensing from above.

The measurable quantity is the reflection coefficient or reflectivity, which is the ratio of the reflected sound intensity and the incident sound intensity. Reflectivity depends on the angle of incidence and on a quantity called the complex ground impedance. Soil is a porous material comprised of a combination of clay, silt and sand particles, which are classified by differing average grain diameter [12]. The acoustic reflection properties of soil depend on its porosity, flow resistivity, tortuosity, steady-flow shape factor, and dynamic shape factor [9]. Porosity and flow resistivity are considered the most important soil characteristics that affect acoustic impedance. At sound frequencies $f$ below $1 \mathrm{kHz}$ the flow resistivity has the largest effect on impedance, and above $1 \mathrm{kHz}$ the porosity is more evident [13]. The acoustic flow resistivity is a measure of the air space volume in the soil near the surface with sound propagation being limited to around $10 \mathrm{~mm}$ [14].

The near-surface nature of the acoustic interaction is ideal for the purposes of sensing the presence of free water on the soil surface. The higher flow resistivity and lack of porosity of water means that it has a higher acoustic impedance than soil. The acoustic impedance is expected to have a clear increasing trend with a greater moisture content, which will plateau for saturated soil [7], [15-16].

A laboratory study $[9,10]$ indicated that reflectance measurements can potentially give estimates of volumetric water content to $\pm 2 \%$. A field measurement [16] where the moisture content was measured and varied used an acoustic impulse, with the amplitude of reflected sound doubling when the moisture content of grassland was changed from $10 \%$ to $35 \%$. The variation in reflectance was found to vary substantially with position once the soil was wetted. There also appeared to be strong sensitivity to the air volume. The studies described above suggest that acoustic reflectance could be a useful tool for remotely sensing free water.

\section{Methods and Instrument Design}

The design of a suitable acoustic sensing system was guided by initial model predictions and by proof-of-concept laboratory measurements. Technical details of the design are provided in Appendix A.

\subsection{Model Predictions of the Reflection from Soil and Water}

Reflectivity from soil was estimated by a model for the acoustic impedance of a ground surface [11]. Typical values of the model's parameters are soil porosity $=0.3$, tortuosity $=1.35$, flow resistivity $=10$ to $10^{3} \mathrm{kPa} \mathrm{s} \mathrm{m}^{-2}$, and pore shape factor ratio $=0.75$. Simulation results are shown in Figure 1 for the frequency range 1 to $4 \mathrm{kHz}$ and for an angle of incidence of $45^{\circ}$. The reflectivity has been normalized to that from soil of porosity 0.5 and flow resistivity $100 \mathrm{kPa} \mathrm{s} \mathrm{m}^{-2}$. 


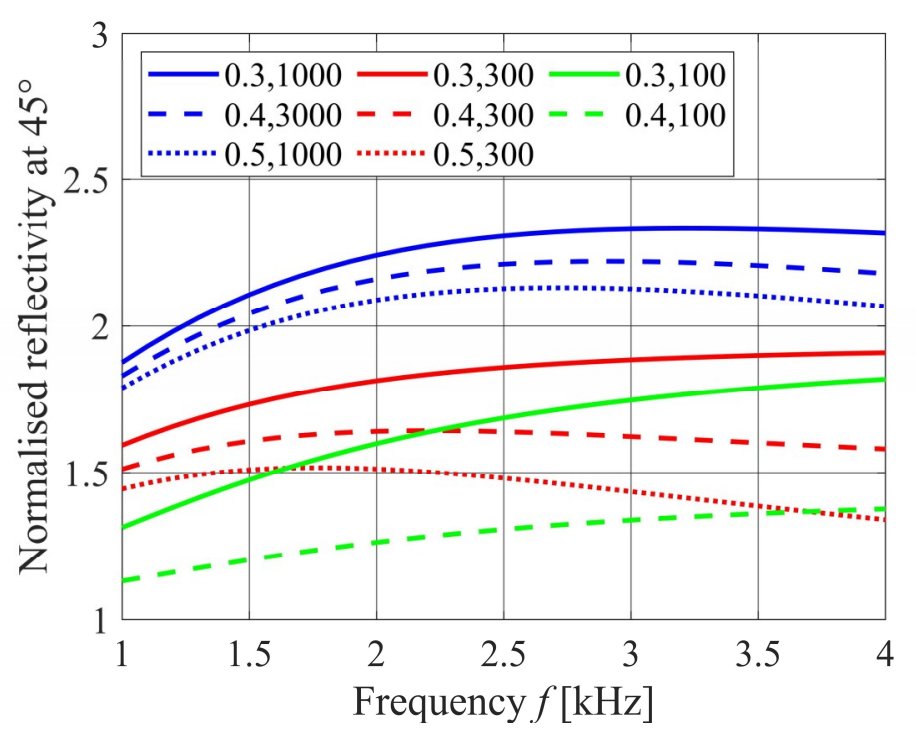

Figure 1. Reflectivity from soil at $45^{\circ}$ incidence normalized to the reflectivity when porosity $=0.5$ and flow resistivity $=100 \mathrm{kPa} \mathrm{s} \mathrm{m}^{-2}$. Porosity and flow resistivity (in $\mathrm{kPa} \mathrm{s} \mathrm{m}^{-2}$ ) are given in the legend.

The greatest fractional variation is predicted to occur at the higher frequencies. An acoustic beam will also be generally sharper at higher frequencies, for a given acoustic antenna size (see Appendix A). Therefore, an acoustic frequency of 2.5 to $4.5 \mathrm{kHz}$ was chosen for the design.

\subsection{Initial Laboratory Investigation}

Initial tests were completed in an anechoic chamber [17]. A tray of dimensions $285 \mathrm{~mm}$ by 385 $\mathrm{mm}$ was filled with $8.6 \mathrm{~kg}$ of dry soil to a depth of $75 \mathrm{~mm}$. Water was added incrementally to the tray to give saturated soil and soil with a water overlayer. Grassed soil was also investigated. A linear array of seven speakers (see Appendix A), directed a short burst of sound onto the tray, which was surrounded by acoustic foam to absorb any sound extending beyond the tray edges. A single microphone $2 \mathrm{~m}$ from the speaker array center recorded sound reflected at $45^{\circ}$. Some results are shown in Figure 2. There is a clear increase in reflectivity with addition of water to the initially dry soil, and the effect of pasture cover appears small in these preliminary tests.

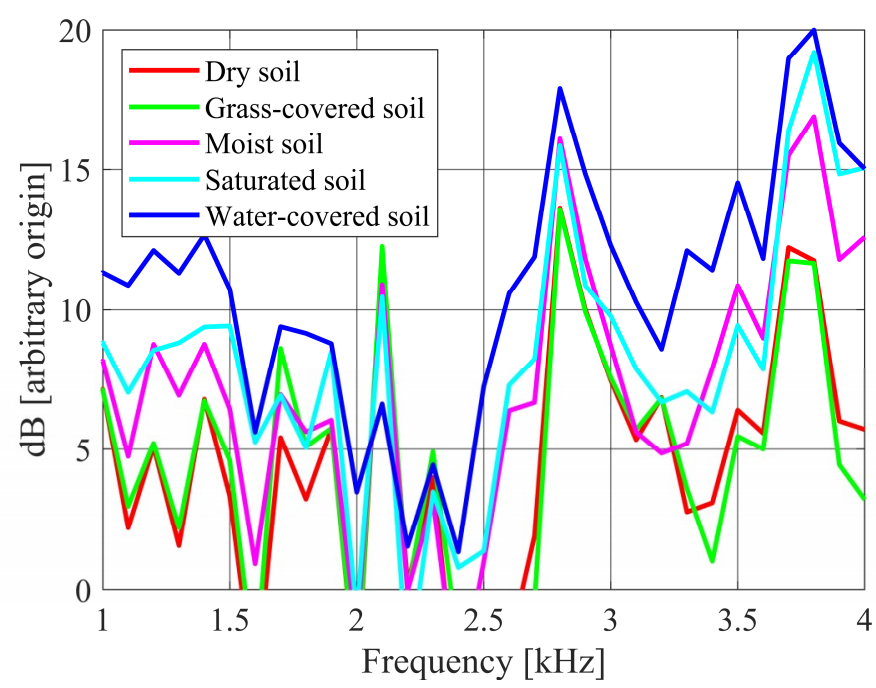

Figure 2. Measured reflection from dry, grass-covered, moist, saturated, and water-covered soil. 


\subsection{Sensing Geometry and Footprint}

The goal is to design an instrument to mount on a large mobile irrigator of the type shown in Figure 3. This means a design height from the ground, $h$, of the acoustic transmitter and acoustic receiver units should be around $2 \mathrm{~m}$ but be flexible.

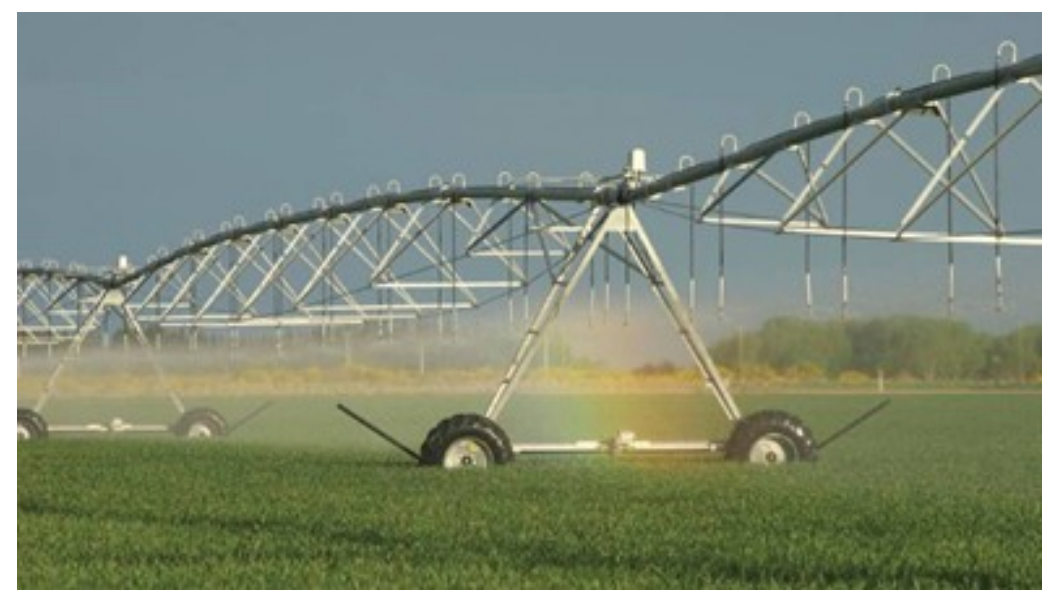

Figure 3. A typical long moving irrigator

With $45^{\circ}$ reflection, the horizontal separation between transmitter and receiver units is $2 h$ (nominally $4 \mathrm{~m}$ ). In order that the reflection angle is sensibly constant, a design angular beam half-width of $5^{\circ}$ was chosen, giving a design half-power 'footprint' of width less than $1 \mathrm{~m}$. Such a design is readily achieved using linear arrays of small speakers and microphones, as described in Appendix A, resulting in a transmitter array and a receiver array, each of length $0.6 \mathrm{~m}$.

Figure 4 shows the predicted footprint sensitivity for recorded intensity, assuming omnidirectional reflection from a rough surface at a frequency of $3.4 \mathrm{kHz}$, with $h=2 \mathrm{~m}$ and with 6 speakers per array. The half-power width is $0.5 \mathrm{~m}$ along the direction between transmitter and receiver, and $2 \mathrm{~m}$ in the transverse direction. The sensitivity falls off rapidly from the central plateau.

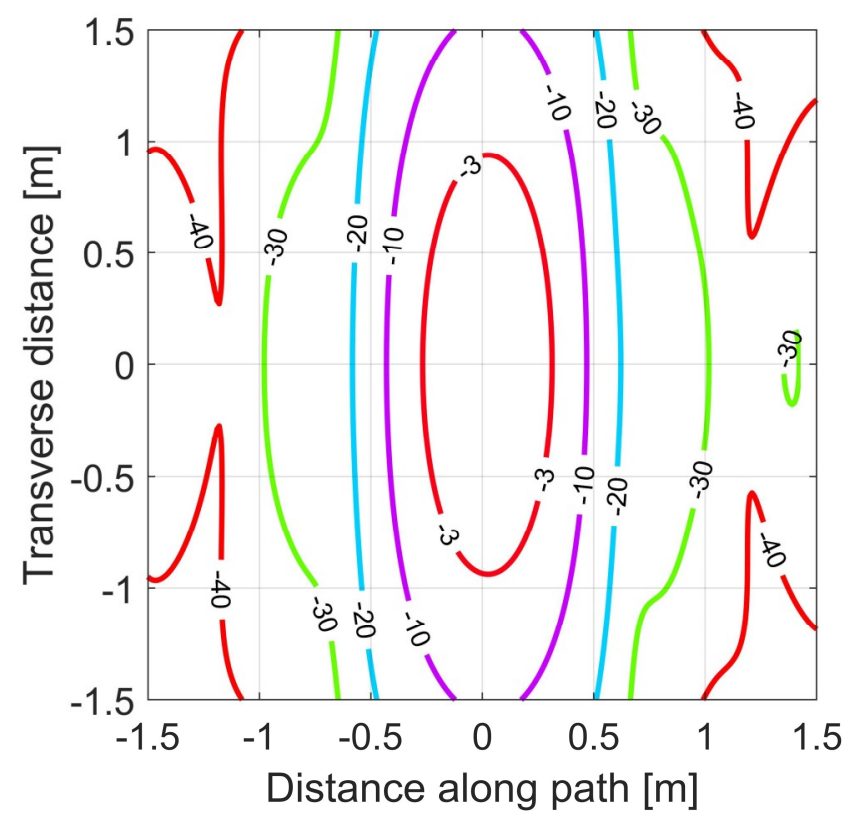

Figure 4. Theoretical footprint sensitivity in $\mathrm{dB}$ for the transmitter and receiver both at a height of $h=$ $2 \mathrm{~m}$, and a frequency of $3.4 \mathrm{kHz}$. 


\subsection{Temporal Resolution}

Although the transmission path, of length $2^{3 / 2} h$ is well-defined with the arrays, there is also a direct-path horizontal transmission of length $2 h$. The path difference of $2\left(2^{1 / 2}-1\right) h$ corresponds to a propagation time difference of $5 \mathrm{~ms}$ for $h=2 \mathrm{~m}$ when the sound speed is $340 \mathrm{~m} \mathrm{~s}^{-1}$. Use of short pulses of duration less than $5 \mathrm{~ms}$ ensures that the direct and reflected signals can be separated. However, in order to also provide robustness against external acoustic noise, such as irrigator spray noise, the short sound pulses are modulated as described in Appendix A. The result of the signal processing of these modulated pulses is to obtain an effective 'compressed' pulse duration of 0.065 $\mathrm{ms}$, or a range resolution of $22 \mathrm{~mm}$.

\subsection{Electrical design}

For design and testing, the speakers were driven in parallel by a laptop-generated modulated signal via a $30 \mathrm{~W}$ audio amplifier. The microphones, also connected in parallel, were sampled by a data acquisition system controlled by the laptop. The system was powered by a battery, which can be maintained via a solar panel. For final operational use something like a Raspberry PI, with an appropriate acoustic add-on module, would be adequate.

\section{Field Measurements}

\subsection{Measurements on Soil Trays}

Field experiments was performed using a $0.45 \mathrm{~m} \times 0.6 \mathrm{~m}$ tray of soil placed at ground level at the center of a frame upon which instrumentation was mounted (see Figure 5). A manometer allowed the water level to be continuously varied. A downward facing 3D structured light camera (Intel RealSenseTM Depth Camera D415) viewed the earth-filled tray, so that topology and pooled water could be recorded. Processing of the 3D camera images allowed the soil surface topography to be quantified, which was of particular use when depressions were manually introduced and allowed to gradually fill with water (see Figure 6).

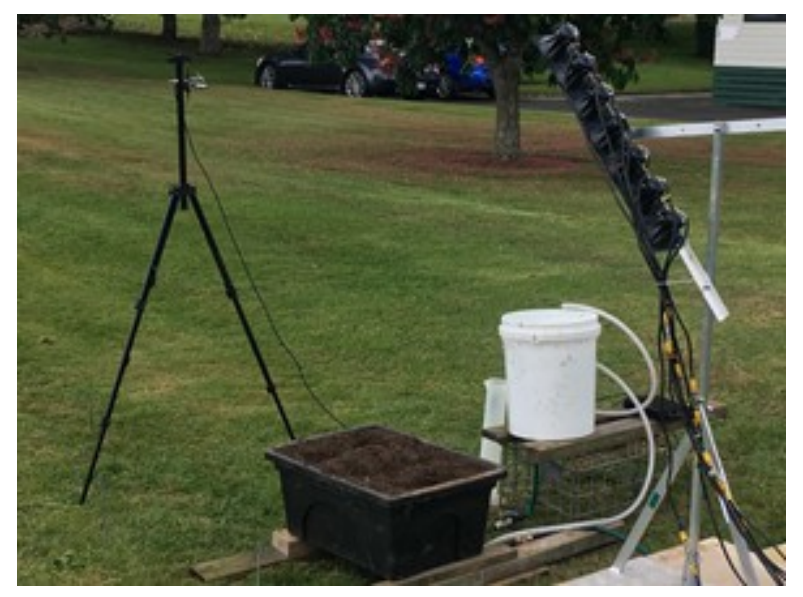

Figure 5. An initial experiment showing the transmitter array mounted at $45^{\circ}$ to the horizontal above a soil tray. A single microphone was used in this experiment. The water level in the soil tray could be adjusted using a manometer.

In the first set of experiments the fraction $\alpha$ of the soil surface covered in water was estimated from 3D camera images and compared with measured acoustic reflectance measured using pulses of frequency 2.5, 2.9, 3.3, 3.7, 4.1, and $4.5 \mathrm{kHz}$ modulated as described in Appendix A. Depressions were manually made in the soil surface and gradually filled with water. Figure 7 shows data from two different experiments. The first, shown in black, includes error bars calculated from the 
variation with frequency at each areal coverage. Results, when normalized to the maximum intensity at each frequency were nearly independent of frequency. The curve in red on Figure 7 shows a much more extensive range of area coverage of water. The salient feature of these experiments is that there is a substantial, statistically significant, increase in reflectance up to a fractional cover of $40-60 \%$ and dropping off thereafter.

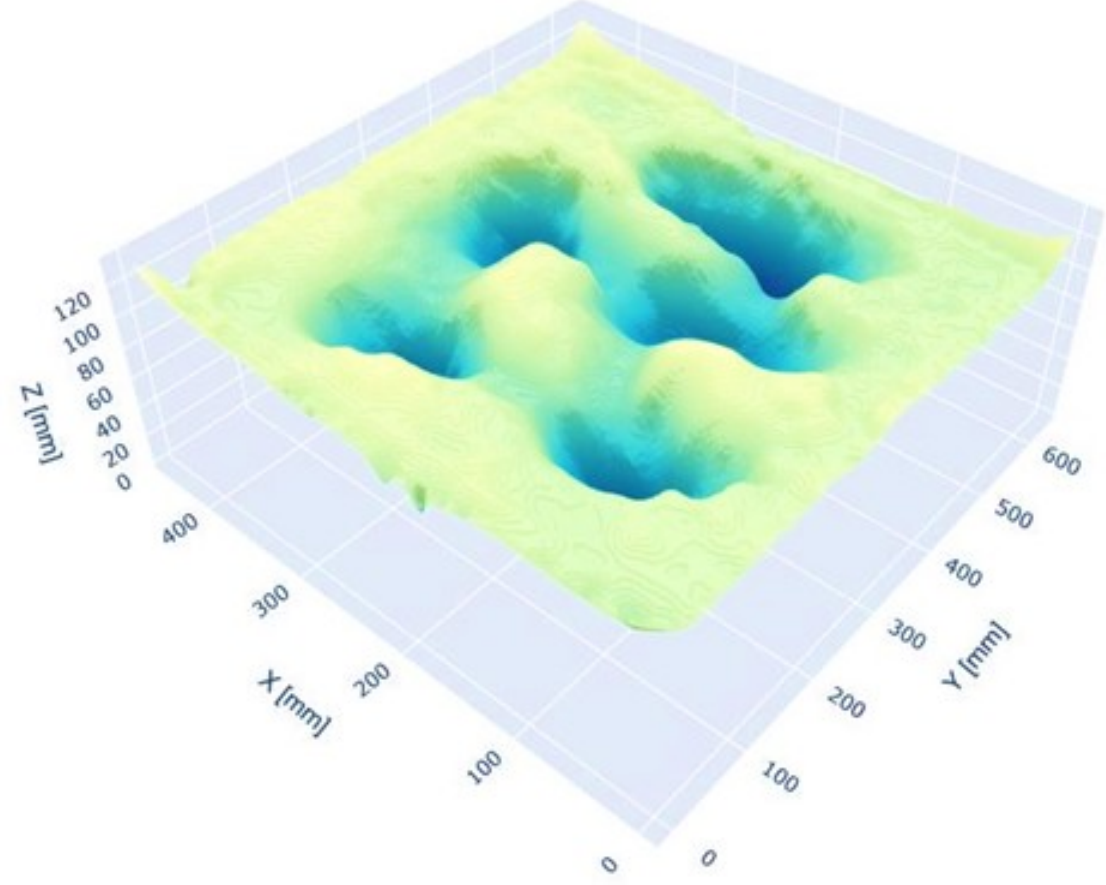

Figure 6. A typical soil surface map from a soil tray experiment.

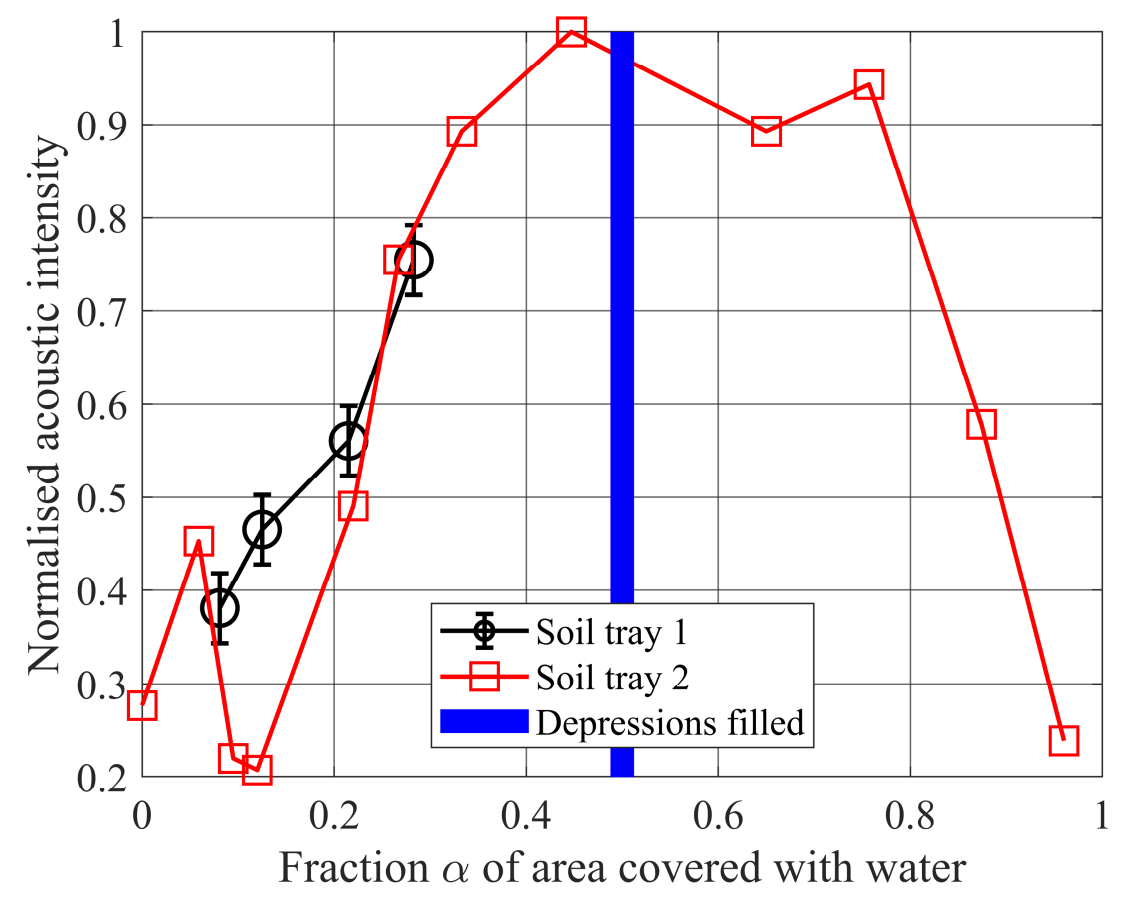

Figure 7. Results from two experiments on wetted soil trays. The fraction of soil surface covered with water is estimated from 3D camera images. The black curve also shows error bars based on the standard deviation of intensities measured at each of the 6 transmission frequencies. The blue bar indicates the point at which depressions became full. 
The sensor was further tested in a field setting. The set up comprised the speaker and microphone arrays, both mounted at $45^{\circ}$ above a bounded runoff plot $(1 \mathrm{~m} \times 1 \mathrm{~m})$. The downward facing 3D structured light camera viewed the bounded runoff plot, so that its topology could be recorded, including any pooled water (see Figure 8). The measurements were progressively done from the soil surface having empty depressions to the state where all the soil surface depressions were completely filled with water. Results are shown in Figure 9, with error bars showing the variability with frequency. The general behavior is similar to that of the two tray experiments, although the peak in this case is at a lower fractional cover $\alpha$.

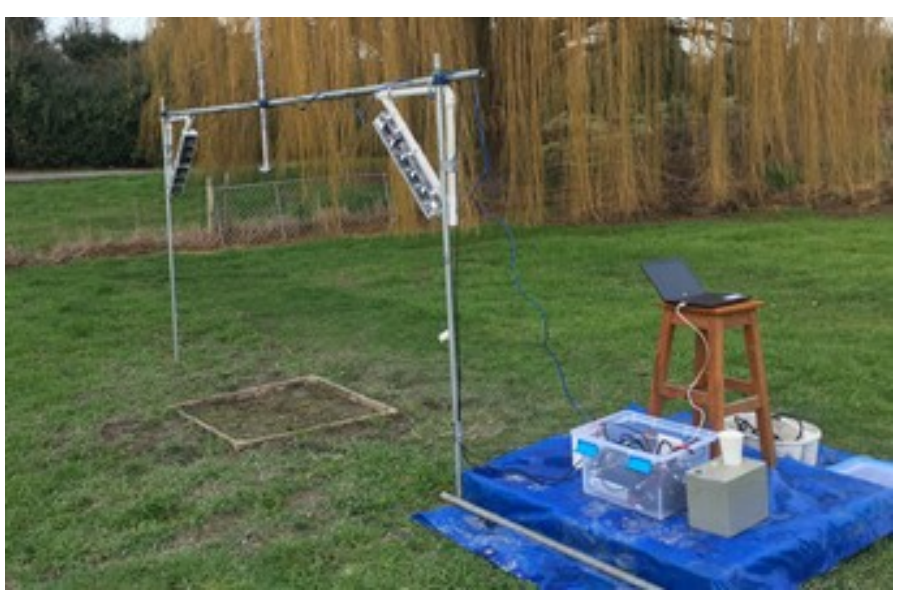

Figure 8. The $1 \mathrm{~m} \times 1 \mathrm{~m}$ bounded runoff plot placed between the transmitter and receiver arrays. The $3 \mathrm{D}$ camera is mounted directly above the runoff plot.

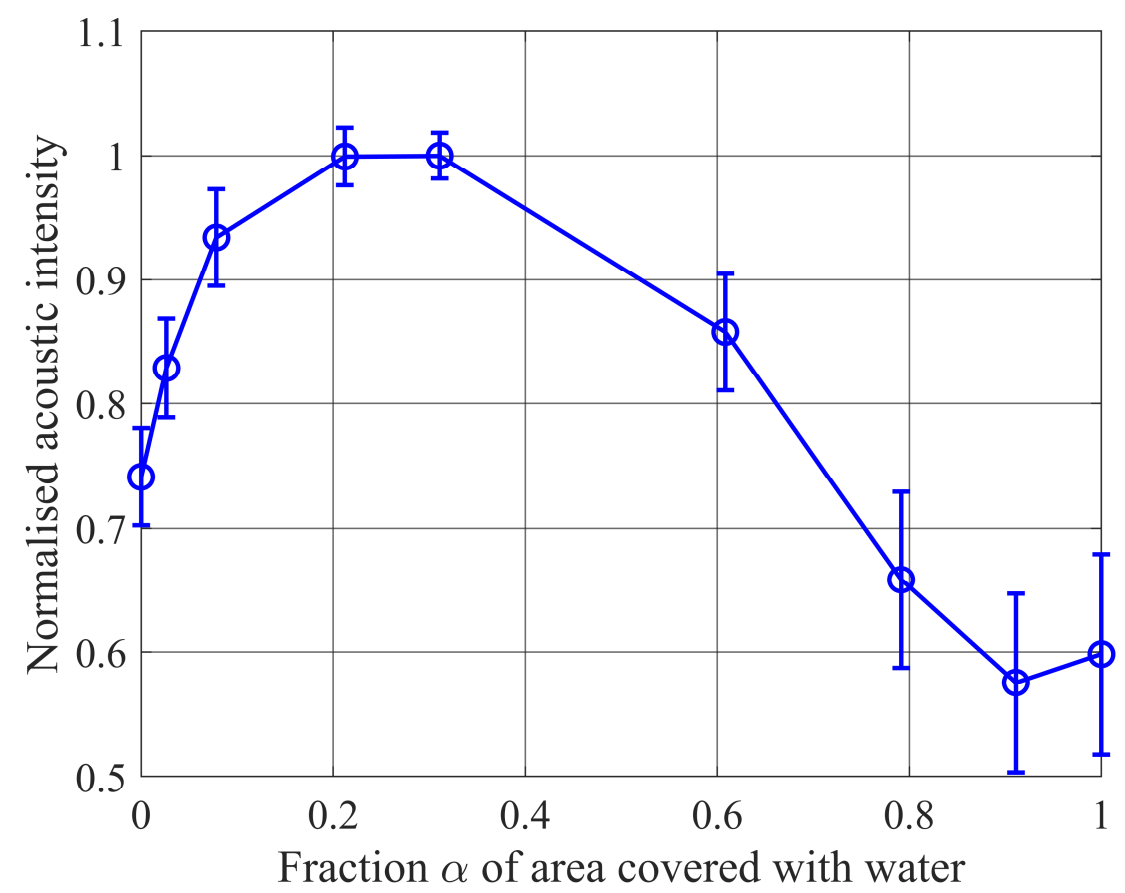

Figure 9. Results from the runoff plot experiment.

\subsection{Measurements Beneath an Irrigator}

Experiments were conducted with the transmitter and receiver arrays mounted on a moving irrigator, and on a frame with the irrigator moving over the frame (Figure 10). Measurements of areal water cover were not possible because of the grass cover, but the soil moisture was monitored at three locations using time domain reflectometry sensors (ML3 ThetaProbe, Delta-T Devices). 
Results are shown in Figure 11 where the error bars are from the variation across the six frequencies transmitted. Although the horizontal scale is different, behavior similar to that of the previous experiments is observed with a significant increase in reflectance up to a soil moisture of around 0.27 $\mathrm{m}^{3} \mathrm{~m}^{-3}$ and a drop thereafter.

\subsection{Sensitivity to a Pasture Canopy}

The ground impedance is known to depend on pasture coverage [11]. In order to evaluate the dependence of reflectivity on pasture cover, the frame shown in Figure 10 was mounted above three different areas of pasture. For each area, reflectance measurements were conducted over uncut pasture, pasture cut to mid-height, and pasture cut to close to the ground. Figure 12 shows results normalized to the 'bare ground' intensities. The biomass dry matter was measured by collecting the cut pasture, drying it and weighing. The biomass is given in $\mathrm{kg}$ per hectare in the figure caption.

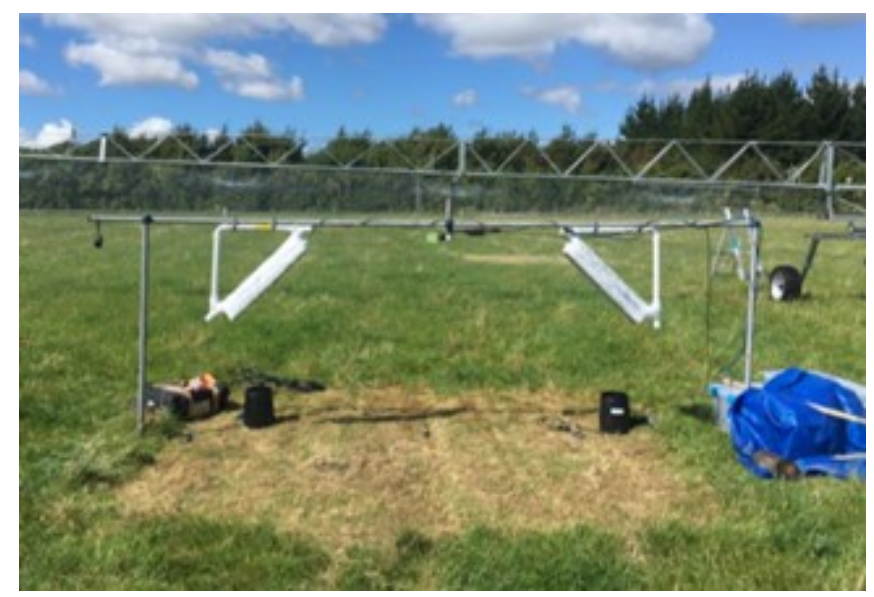

Figure 10. The arrays (white rectangles) mounted on a small frame so that the mobile irrigator can pass over the instrument.

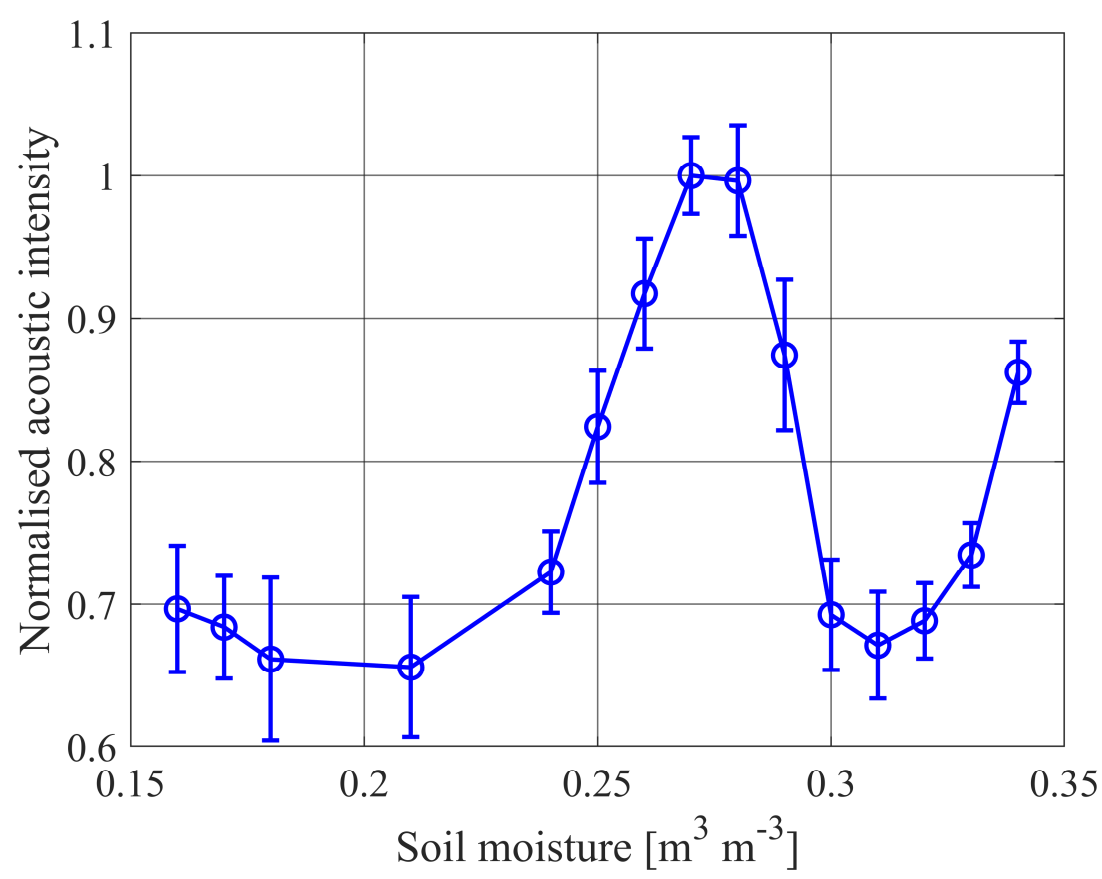

Figure 11. Reflectance measurement dependence on soil moisture. The error bars are from the standard deviation of the measurements across the 6 transmitted frequencies. 
These results indicate a significant pasture effect, although this is not consistent with laboratory observations. There is some concern that the ground may have been compressed during the pasture cutting, and so measurements of pasture effect need to be repeated before the effect can be fully quantified. In addition to the effect of pasture cover, the effect of wetting of the pasture was investigated but found to be negligible.

\section{Discussion}

\subsection{Acoustic Reflectance Model}

The field measurements show a common behavior of an increase in reflectance with increasing water coverage $\alpha$ until a peak is reached. Further increasing water cover leads to a decrease in reflectance. However, modelling reflectance on the basis of changes in porosity as the pores fill, or flow resistivity, shows a monotonic increase in reflectivity with increasing water content. A reason for this discrepancy may be that the traditional reflectance models are generally concerned with large scale sound propagation, whereas in the present case the interest is in only a $1 \mathrm{~m}^{2}$ area in which roughness features are potentially more important.

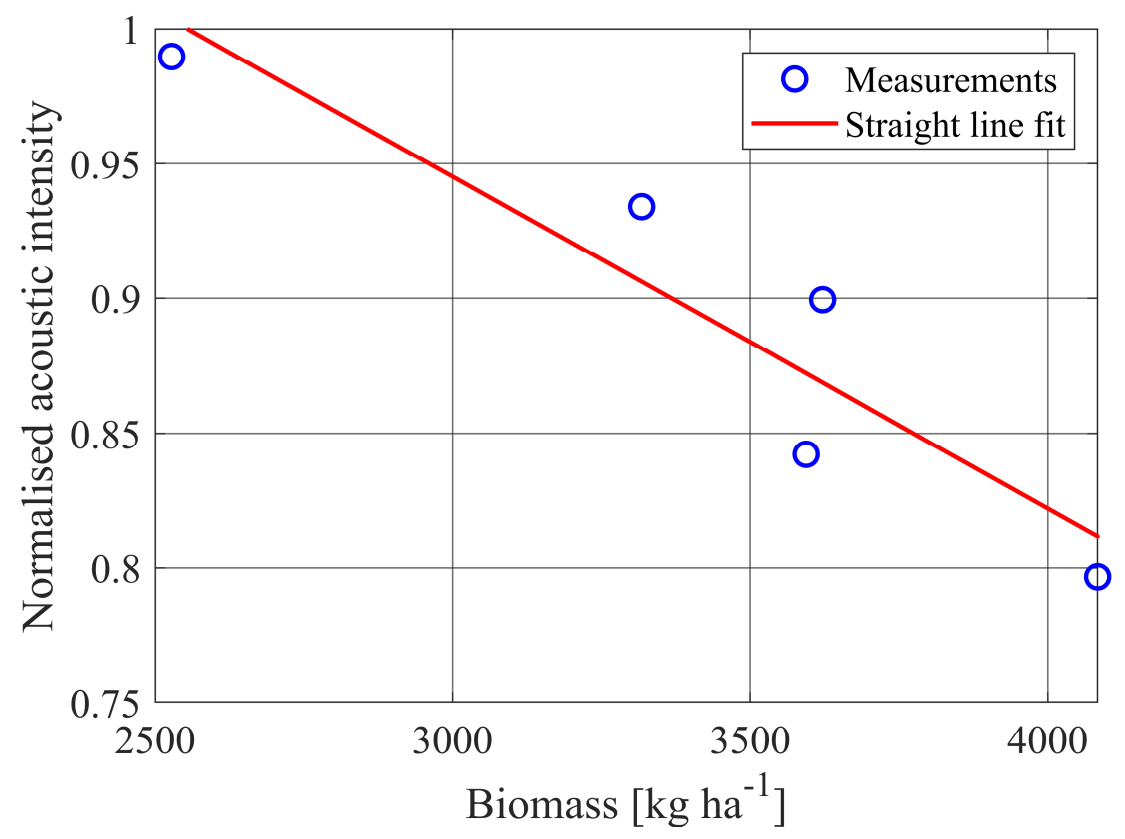

Figure 12. Measured intensities normalized to the bare ground measurements and having different biomass.

Consider a fraction $\alpha$ of the soil surface covered with water. The water is smooth and produces specular (mirror-like) reflection. The fraction (1- $\alpha)$ of rough soil is assumed to reflect more omnidirectionally. The overall reflectivity is

$$
R=(1-\alpha) R_{\text {soil }}+\alpha \gamma R_{\text {water }}
$$

where $R_{\text {soil }}$ is the reflectivity of soil, $R_{\text {water }}$ is the reflectivity of water. The factor $\gamma$ allows for the lower reception of specular reflected sound due to much of the divergent acoustic beam being reflected into angles which bypass the linear array of microphones.

The soil reflectance depends on moisture content and can be written as the sum of a dry soil reflectance term, $R_{d r y}$, and a moisture-dependent term. The moisture-dependent term will increase with water fraction $\alpha$ because of water filling pores. Assume therefore that

$$
R_{\text {soil }}=R_{d r y}+\alpha d R_{\text {soil }} / d \alpha .
$$


Equation (2) can alternatively be considered as a first-order expansion of the dependency of $R_{\text {soil }}$ on $\alpha$. The recorded acoustic intensity can be written as

$$
I=G R \text {. }
$$

The gain $G$, which includes the transmitter and receiver array sensitivities and the angular acoustic beam pattern, can be determined through experimental calibration. Combining (1), (2), and (3), the intensity dependence on water cover will have the form

$$
I=I_{0}+\alpha I_{1}+\alpha^{2} I_{2} .
$$

Results from the two soil tray experiments (shown in Figure 7) and from the runoff experiment (Figure 9) are reproduced in Figure 13 together with quadratic regressions based on the simple model in equation (4). The quadratic regression shown in blue has a rms error of 0.17 and a coefficient of variation $R^{2}=0.65$. The quadratic regression shown in red has a rms error of 0.08 and a coefficient of variation $R^{2}=0.83$. The rms error for the red curve compares closely with the inherent mean measurement error of 0.05 shown in Figure 9. This implies that the simple model fully describes the observed behavior, to within measurement uncertainty.

The two regression curves in Figure 13 suggest quite different dry soil reflectivity. This is possibly because one regression curve was for when a tray was manually filled with soil, and the other curve was from a runoff plot experiment obtained under natural conditions.

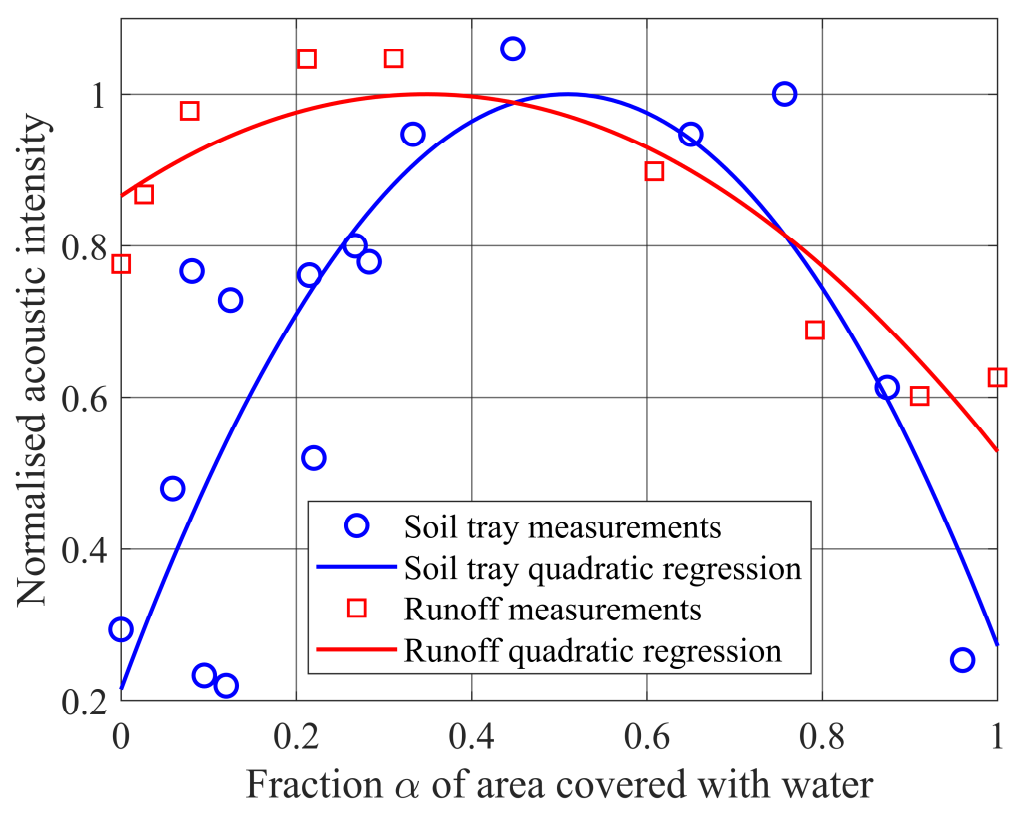

Figure 13. Reflectance measurements from Tray 1 and Tray 2 together with quadratic regressions based on the model.

\subsection{Operational implementation}

The initiation of overland flow generally occurs at a critical value of connectivity, COF, the proportion of the soil surface that is connected via a water-filled pathway to an exit point of the field. Quantifying the development of COF during an irrigation event, therefore, is key to predicting the initiation of overland flow.

The experimental results and model described above relate observed acoustic reflectivity to the fraction $\alpha$ of the soil surface covered with water. Ghimire et al. 2020 [18] used a ponding and redistribution model to relate COF to $\alpha$. They found that the average $\alpha$ required for establishing full hydrological connectivity increases with the increase in depressional storage capacity, indicating a significant link between $\alpha$ and overland flow connectivity. 
The fraction of the soil surface covered with water, $\alpha$, and hence COF can be estimated in real-time from acoustic reflectivity measurements based on inverting (4):

$$
\alpha=-\left\{\left[I_{1} /\left(2 I_{2}\right)\right]^{2}+\left(I-I_{0}\right) / I_{2}\right\}^{1 / 2}-I_{1} /\left(2 I_{2}\right) .
$$

From (5) the estimated uncertainties in regression parameters $I_{0}, I_{1}$, and $I_{2}$, together with the uncertainties in measurements $I$ shown in Figure 7 and Figure 9, can be used to estimate the uncertainty in real-time estimates of $\alpha$. A conservative pulse repetition rate of $8 \mathrm{~s}^{-1}$ was used, with averaging of the acoustic signals over the duration of each $\alpha$ estimate. More realistically the pulse transmission rate could be 100 pulses per second, giving improved estimation of $\alpha$, and hence CoF, from (4). Figure 14 shows the uncertainty $\sigma_{\alpha}$ in real-time operational estimation of $\alpha$ based on these considerations, as acoustic intensity increases with increasing irrigation. For example, from the runoff case in Figure 13 when $\alpha=0.3$, the estimation uncertainty would be $\sigma_{\alpha}=0.086$ or around \pm $30 \%$ of the true $\alpha$. While this is quite a large uncertainty, the real-time decision making would be expected to be based on the trend of COF estimates which would smooth estimation variations.

\section{Conclusions}

A relatively simple acoustic remote sensing instrument has been designed to allow real-time estimation of the extent of free surface water under an irrigation system. The instrument comprises two linear arrays, one for transmission and one for reception. The directionality of the arrays gives a well-defined footprint area on the ground beneath the instrument. The instrument has been mounted on a moving irrigator and can be solar powered and autonomous.

Laboratory and field measurements show that the measured acoustic reflectance increases in a statistically significant fashion up to the point where the fraction of surface covered with water is around 0.5 . For further injection of water, the reflectivity decreases. A simple model is proposed to account for this observed behavior. It is proposed that the decrease in reflectivity is related to the change from diffuse reflection to specular reflection as the fraction of water surface increases. This model agrees with observations to within the measurement uncertainties and has the potential to be developed so that the remote acoustic method can estimate surface free water for real-time irrigation control. There are effectively only three model parameters. These allow for an estimation of the point at which peak free water will occur. The instrumentation combined with the physical model provides the potential for real-time monitoring and control of irrigation systems to mitigate overland flow.

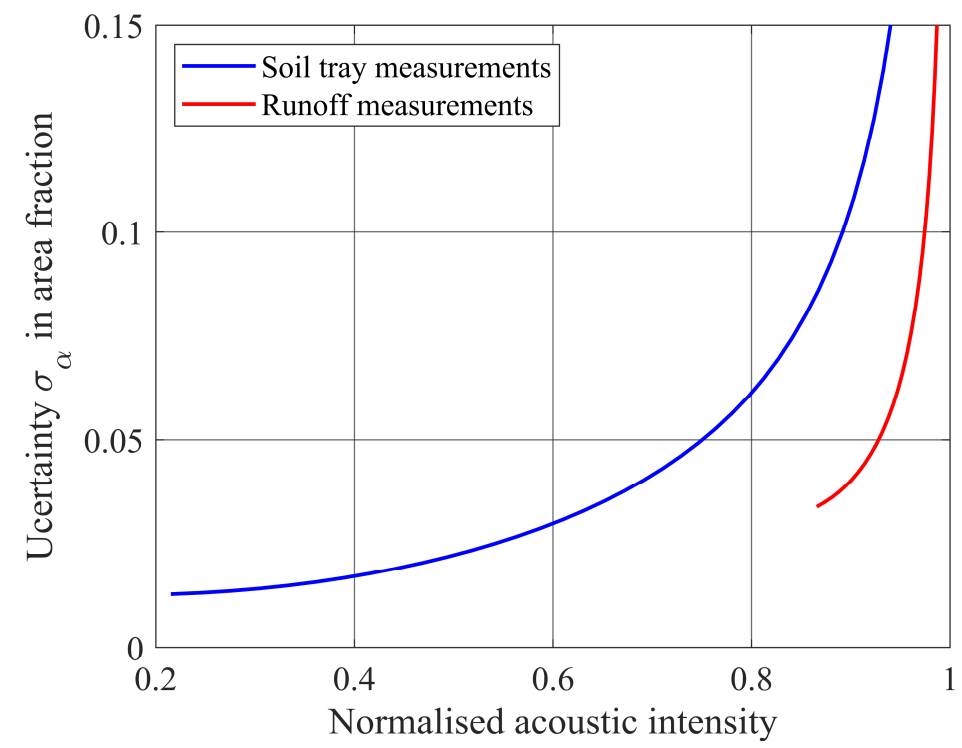

Figure 14. Uncertainty in estimation of $\alpha$ for real-time operational monitoring of CoF.

\section{Patents}


Snow, V., Bradley, S. G., Ghimire, C. P. Ground Surface Condition Sensing In Irrigation Systems.NZ750742, 15 February 2019 NZ754059, 30 May 2019. AgResearch/University of Auckland.

Author Contributions: Conceptualization, Stuart Bradley and Val Snow; Formal analysis, Stuart Bradley, Chandra Ghimire and Laura Grundy; Funding acquisition, Stuart Bradley and Val Snow; Investigation, Stuart Bradley and Chandra Ghimire; Methodology, Stuart Bradley and Chandra Ghimire; Project administration, Val Snow; Resources, Stuart Bradley, Chandra Ghimire and Val Snow; Software, Stuart Bradley, Anna Radionova, Chandra Ghimire and Laura Grundy; Supervision, Stuart Bradley and Val Snow; Validation, Stuart Bradley, Anna Radionova, Chandra Ghimire and Laura Grundy; Writing - original draft, Stuart Bradley; Writing - review \& editing, Stuart Bradley, Chandra Ghimire, Laura Grundy, Seth Laurenson and Val Snow. All authors have read and agreed to the published version of the manuscript.

Funding: This research was funded by the New Zealand Ministry of Business, Innovation and Employment via its Endeavour Smart Ideas contract C10X1708.

Acknowledgments: The authors are grateful for comments by Mostafa Sharifi of AgResearch Ltd.

Conflicts of Interest: The authors declare no conflict of interest. The funders had no role in the design of the study; in the collection, analyses, or interpretation of data; in the writing of the manuscript, or in the decision to publish the results.

\section{Appendix A}

\section{Hardware}

An array of six speakers, spaced at $85 \mathrm{~mm}$ centers, were used to generate short tonal pulses of duration $2 \mathrm{~ms}$ directed downward at an angle of $45^{\circ}$. The Motorola KSN1005A super horn piezo tweeters were chosen, producing sound pressure levels of $74 \mathrm{~dB}$ at $2 \mathrm{kHz}$ and $94 \mathrm{~dB}$ at $4 \mathrm{kHz}$, at $1 \mathrm{~m}$ distance when driven with $2.8 \mathrm{Vrms}$. The speakers have dimensions of $d=85 \mathrm{~mm}$.

Signal generation and data acquisition were handled with a Data Translation DT9836 unit with synchronous input and output controlled from a laptop. The DAC drives the six speakers in parallel via a Dayton_Audio_DTA3116HP $30 \mathrm{~W}$ amplifier, and the ADC samples the six 'microphones' in parallel. Power derives from a battery, which can be maintained via a solar panel. Data is sampled at the standard audio rate of 44100 samples per second with a resolution of 24 bit.

\section{Beam pattern}

The diffraction formula for the intensity from a linear array of $M$ speakers spaced at separations $d$ is

$$
I=I_{0}(\theta)\{\sin (M x) /[M \sin (x)]\}^{2}
$$

where $x=k d \sin (\theta) / 2$ for beam angle $\theta$ and $k$ is the wavenumber [19]. The squared term has a half-power half-width of approximately $M x=2^{1 / 2}$. The angular behavior of an individual speaker is described by $I_{0}(\theta)$ which for these speakers and frequencies is approximately proportional to $\cos ^{4}(\theta)$. Based on (A1) the array gave a directional beam of half-power angular width of $6^{\circ}$ at $2.5 \mathrm{kHz}$ and $4^{\circ}$ at $4 \mathrm{kHz}$ which agrees closely with the measured response, as shown in Figure A1.

The 'far field' region (Fresnel parameter $>1$ ) for this antenna is beyond a range of about $1 \mathrm{~m}$ at a frequency of $4 \mathrm{kHz}$, so the beam is well-formed at the ground.

The KSN1005A speakers are also used as the 6 elements in the microphone array, since a voltage is developed across a piezo crystal in response to the pressure fluctuations from an acoustic wave. The angular response when used as a microphone is the same as when used as a speaker.

\section{Modulation}

In order to provide better performance in the presence of acoustic noise, the transmitted acoustic pulses were phase-encoded with a 13-bit Barker code. The phase changes per-cycle means each pulse is 13 cycles of the base frequency, and pulse durations are $6.5 \mathrm{~ms}$ for $2 \mathrm{kHz}$ to $3.25 \mathrm{~ms}$ for $4 \mathrm{kHz}$. The reflected signal is cross-correlated (or matched filtered) with the Barker code pulse, and 
squared, to give a temporal resolution of $0.13 / f$ or $0.065 \mathrm{~ms}$ at $f=2 \mathrm{kHz}$, so extending the pulse duration past $5 \mathrm{~ms}$ is not a problem. Figure A2 shows results from a field experiment for which $h=$ $2.22 \mathrm{~m}$ and $f=4.5 \mathrm{kHz}$. The matched filter output of the direct, non-reflected, signal is negligible and, in any case, well separated in time from the reflected signal, and the signal-to-noise ratio (SNR) is 39 $\mathrm{dB}$.

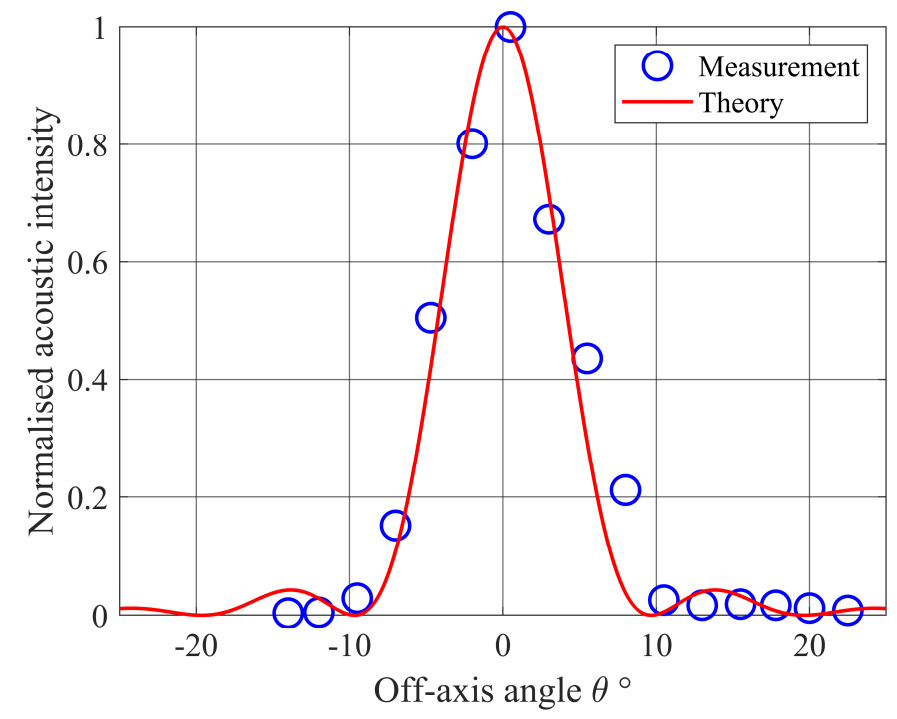

Figure A1. Measured beam pattern at $3.4 \mathrm{kHz}$ compared with theoretical expectations.

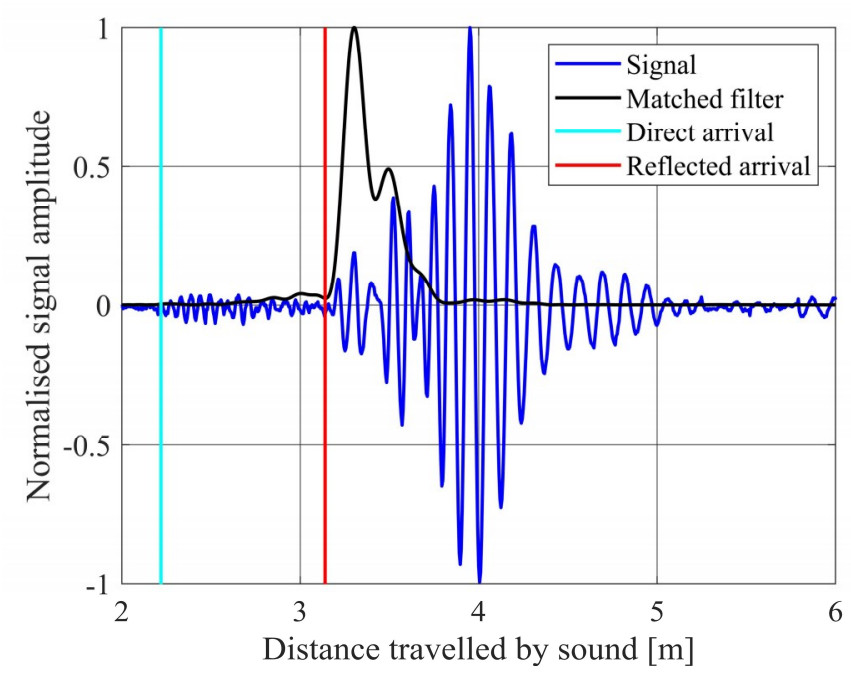

Figure A2. An example of Barker chirp signal analysis.

\section{References}

1. Searchinger, T. Creating a sustainable food future. World Resources Report., Washington DC, USA, 2019. 564pp. ISBN: 978-1-56973-963-1 Library of Congress Control Number: 2019907466.

2. Xingming, Z.; Tao, J.; Xiaofeng, L.; Yanling, D.; Kai, Z. The temporal variation of farmland soil surface roughness with various initial surface states under natural rainfall conditions. Soil Tillage Res. 2017, 170, 147-156.

3. Moreno, G. R.; Alvarez, D. M. C.; Requejo, S. A.; Delfa, V. J. L.; Tarquis, A. M. Multiscaling analysis of soil roughness variability. Geoderma. 2010, 160, 22-30. 
4. Yang, J.; Chu, X. A new modeling approach for simulating microtopography dominated, discontinuous overland flow on infiltrating surfaces. Adv. Water Resour. 2015, 78, 80-93.

5. Chu, X.; Yang, J.; Chi, Y.; Zhang, J. Dynamic puddle delineation and modeling of puddle-to-puddle filling-spilling-merging-splitting overland flow processes. Water Resour. Res. 2013, 49, 3825-3829.

6. Appels, W.M.; Bogaart, P.W.; van der Zee, S.E.A.T.M. Influence of spatial variations of microtopography and infiltration on surface runoff and field scale hydrological connectivity. Adv. Water Resour. 2011, 34 (2): 303-313. doi: 10.1016/j.advwatres.2010.12.003.

7. Cramond, A. J.; Don, C. G. Reflection of impulses as a method of determining acoustic impedance. J. Acoust. Soc. Am, 1984, 75, 382-389.

8. Laranja, R. A.; Budel, V. M.; Amorim, H. J.; Sobczyk, S., Mario, R. Experimental Evaluation of the Acoustic Impedance of Different Ground Types by Sound Propagation in Open-Air Environments. Advanced Materials Research, Trans. Tech. Publ., 2014, 185-191.

9. Horoshenkov, K. V.; Mohamed, M. H. A. Experimental investigation of the effects of water saturation on the acoustic admittance of sandy soils. J. Acoust. Soc. Am., 2006, 120, 1910- 1921.

10. Mohamed, M. H. A.; Horoshenkov, K. V. Airborne acoustic method to determine the volumetric water content of unsaturated sands. J. Geotech. geoenvironmental Eng., 2009, 135, 1872-1882.

11. Attenborough, K.; Bashir, I.; Taherzadeh, S. Outdoor ground impedance models. J. Acoust. Soc. Am., 2011, 129, 2806-2819.

12. Tarboton, D. In Soil Properties. Chapter 4. Utah State University, USA, 2003.

13. Taraldsen, G.; Jonasson, H. Aspects of ground effect modeling. J. Acoust. Soc. Am. 2011, 129, 47-53.

14. Brandão, E.; Lenzi, A.; Paul, S. A review of the in-situ impedance and sound absorption measurement techniques. Acta Acust. united with Acust. 2015, 101, 443-463.

15. Adamo, F.; Andria, G.; Attivissimo, F.; Giaquinto, N. An acoustic method for soil moisture measurement. IEEE Transactions on Instrumentation and Measurement, 2004, 53, 891-898.

16. Cramond, A. J.; Don, C. G. Effects of moisture content on soil impedance. J. Acoust. Soc. Am. 1987, 82: 293-301.

17. Radionova A.; Ghimire C.P.; Grundy L.; Laurenson S.; Bradley S.; Snow V. Acoustic remote sensing for irrigation systems control in agriculture, Proceedings of the 23rd International Congress on Acoustics, Aachen, Germany. 2019, pp. 2870-2877.

18. Ghimire, C. P.; Snow, V.; Bradley, S. G.; Grundy, L. Proximal remote sensing to quantify plot-scale overland flow connectivity. Proc. EGU General Assembly 2020, https://doi.org/10.5194/egusphere-egu2020-11751.

19. Rossing, T. Springer handbook of acoustics, Publisher: Springer Science \& Business Media. 2007. 